\title{
VERS UNE SOCIÉTÉ INCLUSIVE : DES LIENS NÉCESSAIRES ENTRE FORMATION, PRATIQUE ET RECHERCHE
}

\author{
Serge Thomazet et Corinne Merini
}

I.N.S.H.E.A. | « La nouvelle revue - Éducation et société inclusives »

2019/1 N 85 | pages 103 à 120

ISSN 2609-5211

ISBN 9782240049063

Article disponible en ligne à l'adresse :

https://www.cairn.info/revue-la-nouvelle-revue-education-et-societeinclusives-2019-1-page-103.htm

Distribution électronique Cairn.info pour I.N.S.H.E.A..

(C) I.N.S.H.E.A.. Tous droits réservés pour tous pays.

La reproduction ou représentation de cet article, notamment par photocopie, n'est autorisée que dans les limites des conditions générales d'utilisation du site ou, le cas échéant, des conditions générales de la licence souscrite par votre établissement. Toute autre reproduction ou représentation, en tout ou partie, sous quelque forme et de quelque manière que ce soit, est interdite sauf accord préalable et écrit de l'éditeur, en dehors des cas prévus par la législation en vigueur en France. Il est précisé que son stockage dans une base de données est également interdit. 


\title{
Vers une société inclusive : des liens nécessaires entre formation, pratique et recherche
}

\author{
Serge THOMAZET \\ Laboratoire ACTé \\ Université Clermont Auvergne \\ Corinne MÉRINI \\ Laboratoire ACTé \\ Université Clermont Auvergne
}

Résumé: La transformation de nos sociétés par la conception d'environnements durables, inclusifs et accessibles, engage l'ensemble des professionnels, les personnes et leurs familles. Une visée d'accessibilité universelle nécessite des changements de paradigme tant au niveau de l'exercice des métiers, de la fonction des dispositifs d'accompagnement, qu'en matière de formation ou de recherche. Nous avons participé à une expérimentation visant à faciliter le travail des familles autour du projet de vie de leur enfant par la mise en place d'assistants au projet de vie. En travaillant au plus près des professionnels et de leurs employeurs en recherche intervention, nous avons pu identifier de quoi et ce qui faisait ce nouveau métier. Cette forme de recherche a permis d'identifier les ajustements et les mouvements opérés tant par les professionnels que par leurs employeurs; le dispositif de recherche, ouvrant ainsi à d'autres façons de penser la formation.

Mots-clés: Assistant projet de vie - Changement de paradigme - Dilemmes - Formation au travail en intermétiers - Intermétiers - Partenariat - Société inclusive.

Towards an inclusive society: new links between training, practice and research

Summary: The transformation of our societies through the design of sustainable, inclusive and accessible environments engages all professionals and even individuals and their families. Aiming for universal accessibility requires paradigm shifts both in the exercise of professions, the function of support systems, and in training or research. In this context, we participated in an experiment aimed at facilitating the work of families who have to design and support their child's life project, so we focused on studying an emerging profession, that of life project assistant. By working as closely as possible with professionals and their employers in intervention research, we were able to identify what and what made the job possible. We show in the text the adjustments and movements made by everyone (professionals and employers), including in the way research is done, thus opening up new ways of thinking about training.

Keywords: Dilemmas - Inclusive society - Inter-professions - Life project assistant - Paradigm shift Partnership - Training for work in the intermediation sector. 
En quelques années, notre compréhension de ce qu'est l'école inclusive s'est largement modifiée. Autrefois limitée à des dispositifs compensatoires destinés aux enfants handicapés, l'école inclusive devient un projet pour l'école, celui d'une école accessible permettant à tous les enfants une scolarité réussie. Ce projet s'insère dans une finalité plus générale de transformation de nos sociétés par la conception d'environnements durables, inclusifs, pensés dans une logique d'accessibilité universelle. Une telle approche engage l'ensemble des professionnels du domaine, les familles et les dispositifs spécialisés qui vivent une réorientation de leur activité vers l'accessibilité du milieu ordinaire.

C'est dans ce contexte de développement de l'accessibilité de notre société que nous avons participé à une expérimentation visant à faciliter le travail des familles amenées à concevoir et faire vivre le projet de vie de leur enfant dans une logique inclusive. La recherche que nous avons conduite dans ce cadre est centrée sur l'étude d'un métier en émergence, celui d'assistant au projet de vie. Le présent article nous amènera, dans un premier temps, à préciser pourquoi la mise en œuvre d'une société inclusive nécessite l'engagement des familles et de nombreux acteurs en milieu spécialisé et ordinaire. Dans un deuxième temps, nous montrerons I'utilité de mener des recherches prenant en compte la complexité par une étude conjointe de l'activité des différentes parties prenantes, chercheurs, professionnels et employeurs. Enfin, nous donnerons quelques résultats qualitatifs sur le métier d'assistant au projet de vie qui nous permettront de revenir sur les processus de négociation en jeu entre ces trois catégories d'acteurs avant d'en tirer quelques conclusions sur la construction de dispositifs de formation inclusifs.

\section{CHANGER DE PARADIGME DANS L'ÉCOLE ET AILLEURS}

Nous l'avons souligné à plusieurs reprises à propos de l'école (e.g. Thomazet, 2008), le projet inclusif met l'école en difficulté parce qu'il s'appuie sur des valeurs éthiques fortes et un idéel (Albero, 2010) largement partagé, sans pour autant donner aux professionnels le mode d'emploi pour agir en conséquence. Ces valeurs inclusives relèvent d'un choix de société, elles sont maintenant largement connues et largement acceptées (voir par exemple Le Laidier, 2018 dans le cas de l'école) et nous élargissons ici cette question. Elles amènent à passer d'une logique de la sélection à celle de participation. Autrement dit d'une société qui se mérite à une société qui doit se rendre accessible à tous (Gardou, 2012).

Ce changement de valeurs relève d'un changement de paradigme non pas au sens de l'usage que l'on fait d'une théorie scientifique (Kuhn, 1970), mais d'un ensemble de croyances et de valeurs. Le paradigme normalise, et, en cela, configure d'une certaine manière le point de vue à partir duquel sont regardées les choses. Par exemple, il amène, dans l'école, à passer d'un constat cet enfant n'a pas sa place à l'école à un questionnement comment lui donner une place?

Penser les choses ainsi rend nécessaire, notamment pour les élèves les plus éloignés de l'école de passer d'une logique compensatoire à une logique d'accessibilité (Thomazet, 2017) et donc de prendre en compte la complexité des situations (Ebersold, Plaisance et Zander, 2016). Cette approche nécessite un travail 


\section{DOSSIER}

commun entre professionnels et avec les familles (Kalubi, Detraux et Larivée, 2006), cela suppose des négociations (Strauss, 1992) et des formes de traduction entendues au sens de Callon (1986) c'est-à-dire des ajustements qui permettent la rencontre entre les mondes sociaux. II apparaît alors que la plupart des familles n'ont pas la possibilité d'être de réels partenaires d'une part par méconnaissance des possibilités offertes pour leur enfant, mais aussi par difficulté à prendre place dans les dispositifs de coopération (Hill et Taylor, 2004 ; Lesain-Delabarre, 2012; Maubant et Leclerc, 2008).

C'est avec l'objectif de rendre les coopérations plus équitables (Ebersold, 2012) que le nouveau métier dont nous avons observé l'émergence a pris place.

\section{UN DISPOSITIF INNOVANT ET UNE RECHERCHE INTERVENTION}

NEXEM, principal représentant des employeurs associatifs du secteur social, médico-social et sanitaire, en partenariat avec plusieurs associations locales ${ }^{1}$ a voulu faciliter le parcours des familles concernées par le handicap et a mis en place à titre expérimental un nouveau métier d'Assistants projet de vie (APV)² sur sept sites de notre territoire (Agen, Bergerac, Bordeaux, Charleville-Mézières, Nice, Pau et Toulon). La mission de ces professionnels était d'assister les familles dans la conception et la mise en œuvre du parcours de vie de leur enfant. En fonction des situations et des besoins, cette assistance portait sur la scolarisation, les loisirs, I'hébergement, la vie sociale et professionnelle, dans une logique inclusive. Dans un contexte où il existe de nombreux métiers de la coordination entre métiers (Cieslik, Legendre, de Decker et Berrut, 2013; Nadeau, 1989), cette assistance aux familles, qui leur donne les moyens de piloter le projet de leur enfant, est inédite.

La recherche a été mise en place dans le but d'identifier l'activité effective des APV dans cette situation particulière où ils étaient amenés à inventer leur métier. La recherche qualitative mise en place a nécessité une méthodologie adaptée d'une part à la multiplicité des acteurs, salariés et employeurs et, d'autre part, aux choix d'insérer en temps réel des éléments de résultats intermédiaires dans le dispositif qu'elle étudie afin d'aider au mieux la compréhension de la constitution de ce nouveau métier. De cette manière nous avons contribué à la formation des APV aux côtés des autres formations initiées par l'employeur.

Nous avons eu recours à une analyse qualitative longitudinale sur deux ans (voir figure 2 ci-après), menée en situation de recherche-intervention (Mérini et Ponte, 2008) qui s'appuie sur un collectif de recherche constitué de quatre chercheurs ${ }^{3}$, du groupe de professionnels, et des employeurs, commanditaires du dispositif. L'objectif de la recherche était de décrire ce nouveau métier au travers de l'activité des professionnels qui l'exercent, de leurs gestes professionnels, des outils utilisés

1. Adapei Var-Méditerranée, Papillons Blancs de Bergerac, Trisomie 21 Alpes-Maritimes, Aquitaine, et Ardennes.

2. L'assistant au projet de vie peut prendre différents noms en fonction des lieux où il exerce. Dans le présent document, nous conservons la dénomination APV quel que soit le nom d'usage utilisé localement.

3. Les deux chercheurs ont été aidés par Julie Pironom ingénieure de recherche au laboratoire ACTé et Jean Bélanger Phd. Université du Québec à Montréal, nous les en remercions. 
et des difficultés rencontrées. Enfin, une caractéristique forte de ce que l'employeur souhaite être un nouveau métier est son espace d'intervention aux carrefours de différents mondes (scolaires, médico-social, santé, etc.). Dans ce contexte, nous avons cherché à connaître le travail mené dans cet espace que nous qualifions d'intermétiers (Thomazet, Mérini, Gaime, 2014).

\section{UNE RECHERCHE EN SCIENCES DE L'ÉDUCATION}

Le champ scientifique des sciences de l'éducation, au sens de Mialaret (1997), est celui dans lequel nous inscrivons cette étude. Ce champ autorise une lecture de la complexité de l'objet étudié par la mobilisation de savoirs scientifiques issus de sciences connexes qui aident à sa compréhension. Ici et pour ce qui concerne l'analyse nous avons recours à la psychologie ergonomique et une sociologie qualitative qui prend en compte les stratégies des acteurs et leurs ajustements (entre eux et avec le milieu) dans la logique de la sociologie de la traduction et de l'acteur réseau (Akrich, Callon et Latour, 1988) qui fonde ces ajustements sur la négociation.

Ce cadre nous permet de prendre en compte le point de vue des acteurs en nous appuyant sur un recueil de leur activité manifeste en empruntant des outils méthodologiques à la psychologie ergonomique (Clot, Faïta, Fernandez et Scheller, 2001)), mais aussi à d'autres champs scientifiques comme celui développé par Bronfenbrenner (1979) qui permet de situer les interactions évoquées plus haut dans une logique de proximité/distance ou celle de Mintzberg (1982) qui explore les modes de coordination d'un point de vue managérial. L'étude associe dans le même temps une visée épistémique et une visée transformative (Saujat, 2010).

Du point de vue des employeurs, le métier est reconnu à partir de son inscription sociale, le secteur d'activité est reconnu par des conventions collectives, situé dans une branche professionnelle. Ce qui fait métier selon Strauss (1992), c'est son noyau central partagé par les professionnels qui exercent ledit métier, en cela il va y avoir communauté de gestes, une formation adéquate, le partage d'outils, une activité orientée par une intention semblable. Mais un métier est vivant et c'est par ses écarts et ses ruptures temporaires au noyau qu'il évolue dans le temps et sur les territoires. Ce sont ces distances relatives au noyau qui permet d'observer les mouvements du métier et tenter d'en comprendre le sens. Ainsi, les décisions que prennent les professionnels nous permettent d'en éclairer le sens c'est-à-dire à la fois la signification et l'orientation.

\section{Observer les processus de prise de décisions, l'activité et les stratégies organisationnelles}

Les décisions sont les moments où se confrontent des logiques individuelles (intra et inter personnelles), collectives (inter-organisationnelles) et écologiques dans la prise en compte des contraintes et des ressources locales. Dans la perspective de Bakhtine (1978), les décisions peuvent être assimilées à des unités de temps et des lieux, des "chronotopes " traduisant la configuration sociale et spatio-temporelle 


\section{DOSSIER}

du processus collaboratif. Nous avons donc recours à la sociologie pour saisir les stratégies et contre-stratégies professionnelles à l'œuvre et comprendre, par l'étude du collectif, le métier d'APV.

De son côté, la psychologie ergonomique nous permet de saisir, au-delà de la tâche réalisée ici et maintenant, l'activité, autrement dit "ce qu'on cherche à faire sans succès et qui nous échappe, ce qu'on s'interdit de faire, ce qu'on fait sans vouloir le faire, ce qu'on fait pour ne pas faire ce qu'on nous demande de faire, autrement dit les activités suspendues, empêchées ou, au contraire déplacées " (Clot, 1999, p. 119). L'analyse critique des stratégies, étudiées à différents niveaux de grains, donne à la fois accès à l'activité des professionnels et aux stratégies organisationnelles de ces mêmes professionnels dans leur environnement. Nous nous interrogeons sur la manière dont l'une et l'autre interagissent, impactent l'évolution des pratiques, contribuent à la construction du métier. Enfin, il s'agit pour nous d'explorer les gestes de métiers des APV, les outils qu'ils élaborent, d'observer en quoi et dans quelles perspectives ces éléments se stabilisent et deviennent propres à un métier et le fondent.

Derrière les éléments théoriques qui viennent d'être évoqués, nous assumons les choix suivants:

- Les contextes influencent les processus cognitifs de même qu'en retour les processus cognitifs refaçonnent stratégiquement le contexte (Brönfenbrenner, 1979),

- Les métiers ne sont pas monolithiques et ne sont pas le seul résultat d'une prescription qu'elle soit règlementaire (prescription primaire) ou liée à la formation (prescription secondaire). Les professionnels, en effet, inventent et réinventent leur métier: "L'homme est plein à chaque minute de possibilités non réalisées " (Vygotski, 1925/1994) l'exploration de la décision est donc un point clé de compréhension des métiers. Nous considérons donc les APV et leurs partenaires comme des professionnels stratégiques.

\section{Une recherche engageant une équipe de chercheurs, les employeurs et les professionnels}

Dans notre recherche intervention, trois types d'acteurs sont impliqués: I'équipe de recherche, les employeurs et les professionnels. Chacun a comme préoccupation (Veyrunes, 2011) un métier avec des visées partagées de modélisation, même si les finalités divergent en fonction des intérêts, individuels ou collectifs défendus par chaque partie prenante (Mérini et Bélanger, à paraître).

Le recueil de l'activité des trois acteurs a été mis en place de manière différenciée. L'activité des APV a été recueillie par l'intermédiaire d'un groupe de travail (Caroly et Clot, 2004). Dans le cas des employeurs, nous avons utilisé les réunions institutionnelles ainsi que les documents institutionnels. Enfin, l'équipe de recherche a rendu compte de son travail sous la forme d'écrits (textes ou diaporama).

Pour favoriser à la fois le respect des préoccupations de chacun, protéger les salariés et, dans le même temps, l'indépendance de la recherche, nous avons dissocié le travail de recherche avec les professionnels des échanges plus techniques de gouvernance avec les employeurs. Seuls les résultats validés par un collectif de 
recherche réunissant les salariés et les chercheurs pouvaient être transmis aux employeurs.

Le travail est logiquement situé dans un contexte sociohistorique, politique, géographique et administratif qui peut biaiser les résultats, engendrer des ascendances ou des formes de manipulations faisant valoir des revendications, ou tentant d'imposer des pratiques. C'est au travers de l'existence de ces turbulences, et des négociations unissant les trois univers ceux des professionnels, de la gouvernance et de la recherche, que nous parlerons de partenariat.

L'existence d'un collectif de recherche (AVP et chercheurs) a facilité I'accès aux terrains et à des données se voulant authentiques et instauré un climat de confiance entre les chercheurs et les salariés qui savaient que leur parole était respectée et protégée. Les échanges entre professionnels de cultures et de références différentes, accompagnés par les chercheurs, ont amené à confronter les subjectivités et à objectiver des savoirs propres à l'intermétiers qui ont été mis en débat avec les employeurs. Ce sont ces interdépendances entre les univers et leurs intérêts propres que nous qualifions de complexes au sens de Morin (1977).

En réponse à cette complexité, nous avons croisé plusieurs protocoles pour cerner l'activité d'accompagnement des professionnels qui est tout à la fois orientée par le projet de la famille et du jeune, mais aussi du milieu, et en comprendre les déterminants. Ces protocoles sont détaillés dans la partie suivante.

\section{Opérationnalisation et outils de recueil des données}

Comme nous l'avons dit plus haut, le dispositif de recherche-intervention a été déployé sur sept sites avec plus d'une dizaine d'APV en tout. II intègre, d'une part "les employeurs", regroupés au sein d'un comité technique (administrateurs de la fédération d'employeurs, chargés de projet, consultants, responsables hiérarchiques) d'autre part les dix professionnels APV et enfin des membres de l'équipe de recherche. Dans la figure ci-après le dispositif de recherche, symbolisé par un trait discontinu, est constitué du collectif de recherche et du comité technique.

Figure 1: Organisation collective du travail de recherche

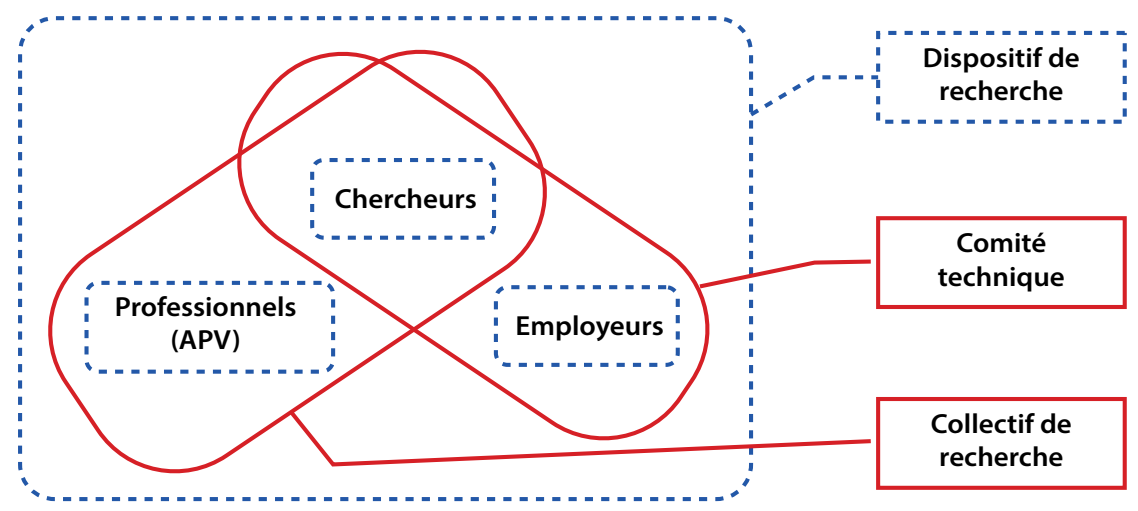




\section{DOSSIER}

Au sein de ce dispositif, nous avions trois objectifs, premièrement situer le métier dans le tissu socioprofessionnel où il s'exerce, deuxièmement saisir la nature de l'activité d'accompagnement des familles et enfin identifier les relations partenariales par lesquelles I'APV travaille le milieu ordinaire pour rendre possible les projets.

Pour situer le métier dans le tissu socioprofessionnel, nous avons utilisé des grilles d'enquête socio-organisationnelle, renseignées par les APV au début de leur activité, puis deux ans plus tard pour saisir la place de ce métier dans le champ professionnel au travers des organigrammes, des modes de financement, des modalités de gouvernance adoptées, mais aussi de la formation et des métiers exercés par les APV. Pour saisir la nature de l'activité, nous avons utilisé:

- Des journaux de bord mensuels construits à partir de catégories négociées avec les APV pour mesurer et quantifier leur activité (temps attribué à l'accompagnement, à la communication, à la bureautique, à la formation / recherche / analyse de pratique...). Ces journaux ont été recueillis sous forme numérique, une analyse qualitative a été faite à partir des tendances relevées.

- Des "boîtes à outils " des APV pour identifier leur activité et l'organiser selon une catégorisation négociée de leurs outils (outils de communication, administrativofinanciers, de suivi des familles, d'accompagnement, d'évaluation, etc.). Les outils ont été analysés collectivement par le groupe de recherche, à partir de classements et de comparaisons.

- Des autoconfrontations simples (3) et croisées (6) à partir de vidéos de situations professionnelles d'accompagnement d'une famille pour saisir la nature de l'activité de I'APV. L'autoconfrontation est une méthodologie qui favorise en entretien l'expression par l'acteur de l'expérience vécue, facilitée par la confrontation de cet acteur à sa propre activité (Flandin, 2017). L'autoconfrontation peut être simple, lorsque les professionnels sont confrontés individuellement à leur activité ou croisée, lorsque les entretiens regroupent plusieurs professionnels (Clot et al., 2001). L'ensemble des enregistrements a été retranscrit et les verbatims analysés par une méthode d'analyse de contenu.

- Nous avons effectué un repérage écosystémique permettant de situer les partenaires de I'APV dans une logique de proximité/distance permettant de voir évoluer leurs interactions dans le temps (T1 début de recherche, T2 6 mois plus tard et en T3 6 mois encore plus tard). Les schémas ont fait l'objet d'une analyse collective en particulier au sujet de l'orientation des échanges et de leur évolution dans le temps.

Ces données sont recueillies sur les différents terrains, soit par les APV (enquêtes, grilles), soit les chercheurs (vidéos de situations professionnelles, autoconfrontations) ou encore lors de journées recherches nationales. Ces journées ont été associées à des temps de formation et à une réunion du comité technique (voir la figure 1) sous une forme ritualisée de la manière suivante: un ou deux jours de formation (concernant juste les APV et les formateurs dépêchés par les employeurs), une journée du collectif de recherche unissant exclusivement les APV et l'équipe de recherche et une journée de réunion du comité technique (chercheurs, employeurs, voir la figure 1). 
Lors de nos journées recherche, nous mettons en discussion dans le collectif les traces recueillies sur les différents terrains, soit en forme brutes (extraits de vidéos, grilles...), soit sous une forme retravaillée par les chercheurs (documents de synthèse de l'activité sur les différents sites, organisation des partenariats, etc. faisant apparaître notre lecture des situations). Cette présentation de traces objectives de l'activité permet la mise en place de débats de métiers, de controverses propices tant à la conscientisation des gestes de métiers qu'à l'identification des marges d'évolution du métier. Lors des rencontres nationales, les modes d'échanges et les calendriers de travail sont ajustés et finalisés en fonction des résultats obtenus et des difficultés rencontrées. Entre ces moments formels de rencontre, nous partageons des outils collaboratifs facilitant les échanges (dossiers partagés, documents partagés, agendas partagés, conférences à distance, etc.) permettant aux APV et aux employeurs de faire remonter leurs données, mais aussi leurs remarques, leur analyse sur les documents produits, et aux chercheurs de diffuser des éléments de résultats et d'analyse en vue de leur validation (par les APV) et d'information (pour le comité technique).

Ainsi, le travail longitudinal de recherche s'est étiré sur deux ans et nous avons procédé à des prises d'information en 2 ou 3 étapes selon les données recueillies. Les techniques méthodologiques diversifiées utilisées apportent, comme nous le souhaitions un éclairage sur le métier à partir de différents points de vue prenant en compte la subjectivité des différents types d'acteurs ce qui permet les ajustements décrits ci-après.

Ces différentes techniques ont été croisées pour valider nos données à la fois par saturation et triangulation des résultats. Les données ont été recueillies par les chercheurs directement auprès des sujets. L'ensemble des résultats a été soumis aux APV pour validation avant diffusion aux employeurs et dans les réseaux de recherche. 

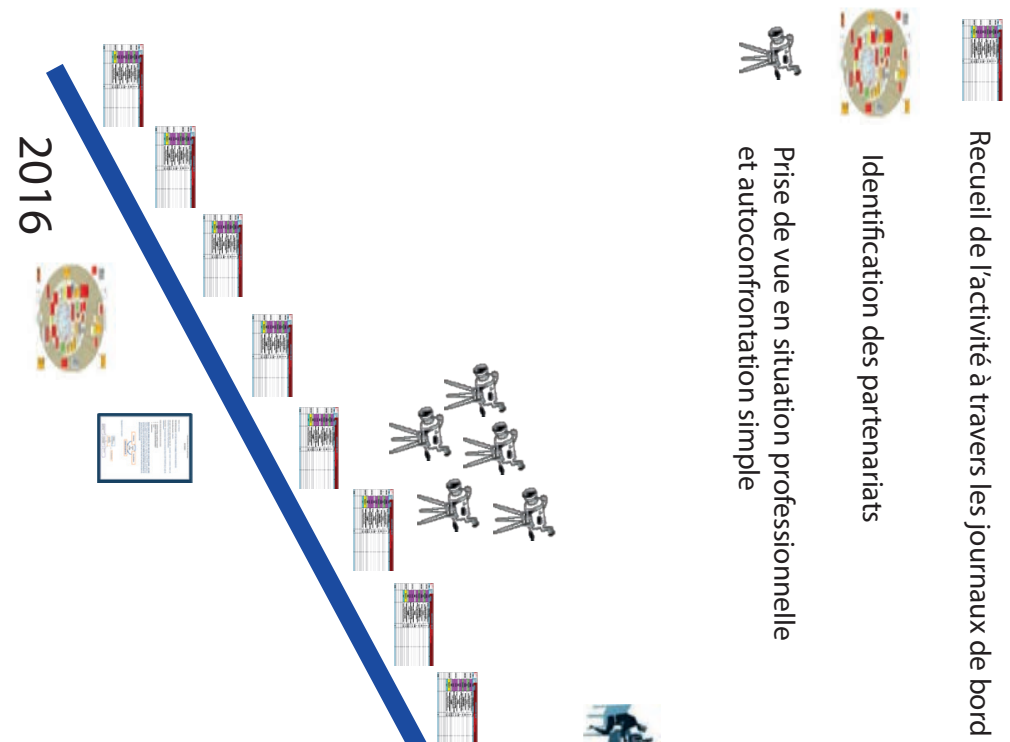

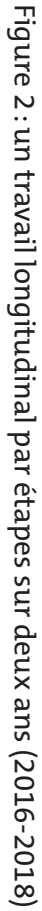
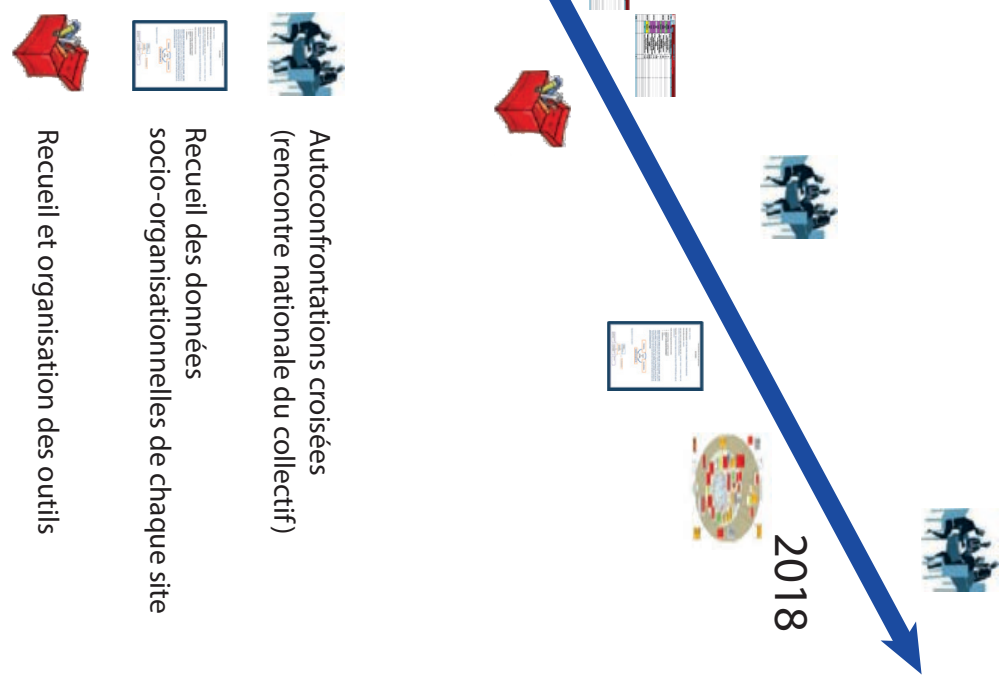


\section{RÉSULTATS}

Nous ne présenterons ici que quelques résultats relatifs au métier. Ce qui nous importe, pour le présent article, c'est de mettre en avant les indices manifestes de changements de paradigmes et de leur partage afin de nourrir la discussion qui suivra et qui portera sur le processus formatif.

\section{Le métier, ce qu'il est et ce qui le fait}

En réponse à la commande qui est de venir en appui des familles, les APV découvrent rapidement à la fois la grande méconnaissance des familles qui repèrent tout à la fois, les démarches complexes qu'elles ont à engager pour obtenir de l'aide et la nécessité de construire des " projets ${ }^{4}$ " de vie pour leur enfant. Les APV ont aussi rapidement découvert que ces projets pouvaient concerner des domaines multiples: scolarisation, hébergement, insertion professionnelle, soin, loisirs, mobilité... Ils ont aussi rapidement pris conscience que, pour faire leur travail, ils doivent aller au-delà de la fiche de poste initiale de I'APV, pour répondre aux besoins des parents et de la fratrie.

La première compétence acquise par les APV est donc législative et réglementaire: I'APV, doit apporter des informations en matière de démarches, de montage de projet ou de droit des personnes handicapées. Pour cela, il travaille avec les familles, et dans le même temps mobilise ou informe le milieu ordinaire sur les capacités du jeune et de sa famille pour promouvoir ou faciliter la création d'espaces et de moments inclusifs.

La dimension éthique de non-guidage est omniprésente pour l'APV: c'est à la famille de faire ses choix et l'APV I'accompagne. Cet aspect du métier est difficile à opérationnaliser lorsque beaucoup de familles demandent avant tout des conseils. L'expertise qu'ils ont développée en réponse à cette demande, dans le respect de leur mission, est de faciliter la montée en compétence des familles et du jeune. Ainsi, sans " conseiller ", en donnant une information complète et objective, ils facilitent les prises de décisions et les choix stratégiques des familles.

Ces professionnels ont rapidement découvert que leur métier nécessitait d'être extrêmement stratégique en ajustant leur action et en composant avec les données du réel et notamment des territoires. Les APV ont donc développé une bonne connaissance des ressources potentielles, matérielles et humaines présentes sur leur territoire, à même de favoriser les projets des familles qu'ils accompagnent. Ils ont construit des réseaux et un "carnet d'adresses " mobilisables en fonction des besoins.

Les APV travaillent à deux niveaux: ils développent des compétences et ont pour tâche d'amener les familles à en faire autant. Par exemple, ils développent des expertises en montage de projets dans de très nombreux contextes (école, loisirs, etc.) et amènent les familles à faire de même par une compréhension du milieu. On le voit, le métier d'APV nécessite des connaissances et compétences leur permettant d'ajuster leur action de manière singulière, en respectant des " fondamentaux du métier " comme la neutralité, l'ouverture des possibles et l'échange. Ils ont eu à développer des principes d'action comme ne pas être intrusif et pourtant soutenir

4. Les mots ou expressions en italiques et entre guillemets sont extraits de nos verbatims. 


\section{DOSSIER}

la famille quand, dans certaines périodes, celle-ci n'est plus en capacité d'agir ou croiser leur regard avec d'autres professionnels.

Ce qui fait le métier, ce sont aussi les outils mis en place, construits avec d'autres professionnels: des fiches pour évaluer les besoins des familles, le carnet d'adresses que nous évoquions plus haut ou encore des documents de suivi des familles. Ces outils, même s'ils ont été largement adaptés aux besoins de I'APV, relèvent cependant de la panoplie de tout professionnel du travail social. II apparaît dans notre recherche que c'est plus dans l'usage des outils que dans les outils eux-mêmes que la spécificité du métier d'APV apparaît.

\section{Des dilemmes à dépasser}

Comme tous les métiers, celui d'APV se structure et se développe autour de dilemmes (Prot, Mezza, Ouvrier-Bonnaz, Reille-Baudrin et Vérillon, 2010) entre des logiques ou des choix stratégiques d'égales valeurs. Le dilemme majeur des APV est de choisir entre accompagner les familles pour leur permettre de trouver des réponses rapides en s'ajustant au milieu ou transformer celui-ci pour le rendre accessible et adapté aux besoins. L'un n'est pas exclusif de l'autre, mais à chaque fois la question se pose pour l'APV. Par exemple, dans le cas d'une recherche d'un accueil de loisirs pour un enfant, deux réponses peuvent être apportées, I'une à court terme et l'autre à plus longue échéance. À court terme, c'est faire avec l'environnement actuel pour une réponse immédiate, mais pas idéale. À long terme, c'est travailler à l'évolution du milieu pour une réponse plus adaptée.

Ces dilemmes ne se règlent pas en une fois, les ajustements se font peu à peu dans le temps et cela engendre des obstacles comme celui de maintenir l'engagement des familles dans la conception et la mise en œuvre de projets sur le long terme. C'est pour cela que le métier d'APV nécessite tout à la fois d'être réactif et d'agir dans le court terme pour apporter une aide rapide, tout en ne perdant pas de vue le long terme et l'évolution du milieu. Un dilemme se dépasse par des pas de côté, des reproblématisations ou des triangulations permettant de positionner la situation autrement.

La recherche a permis d'identifier bien d'autres dilemmes dont nous ne pouvons rendre compte ici et qui feront l'objet d'un autre article.

\section{Ce qui permet au métier de se développer : la dimension transformative du travail de recherche}

Si les dilemmes permettent de faire bouger le métier encore faut-il percevoir les mouvements et les accompagner pour qu'ils soient vecteurs de développement professionnel. Les professionnels dans leurs situations de travail ne perçoivent pas nécessairement les mouvements de leur métier, ils disent: " on a le nez dans le guidon " ou " difficile de se regarder pédaler". La recherche offre aux professionnels une lecture singulière de leur métier et une organisation collective qui permet des échanges et des controverses en vue de leur développement professionnel. Nous allons à présent analyser plus finement un exemple de mouvements que nous avons perçu du métier et mettre en relief le rôle de la recherche intervention dans la montée en compétence des professionnels. La figure 3 ci-après s'appuie sur deux dilemmes, 
liés à la fiche de poste initiale qui donne une unique mission accompagner la famille. Le premier dilemme oppose cette commande d'accompagner la famille, souvent réinterprétée comme accompagner les parents avec la nécessité que ressentent les APV d'accompagner aussi le jeune, pour lequel ils constatent qu'il peut avoir d'autres envies que ses parents, et au-delà, les autres membres de la famille. Le second dilemme met en tension la commande d'accompagner la famille avec la nécessité, que découvrent rapidement les APV, de transformer l'environnement pour trouver des réponses pertinentes.

Figure 3 : accompagner la famille, aider le jeune, ou transformer l'environnement (repris de Mérini, Thomazet et Bélanger, 2018)

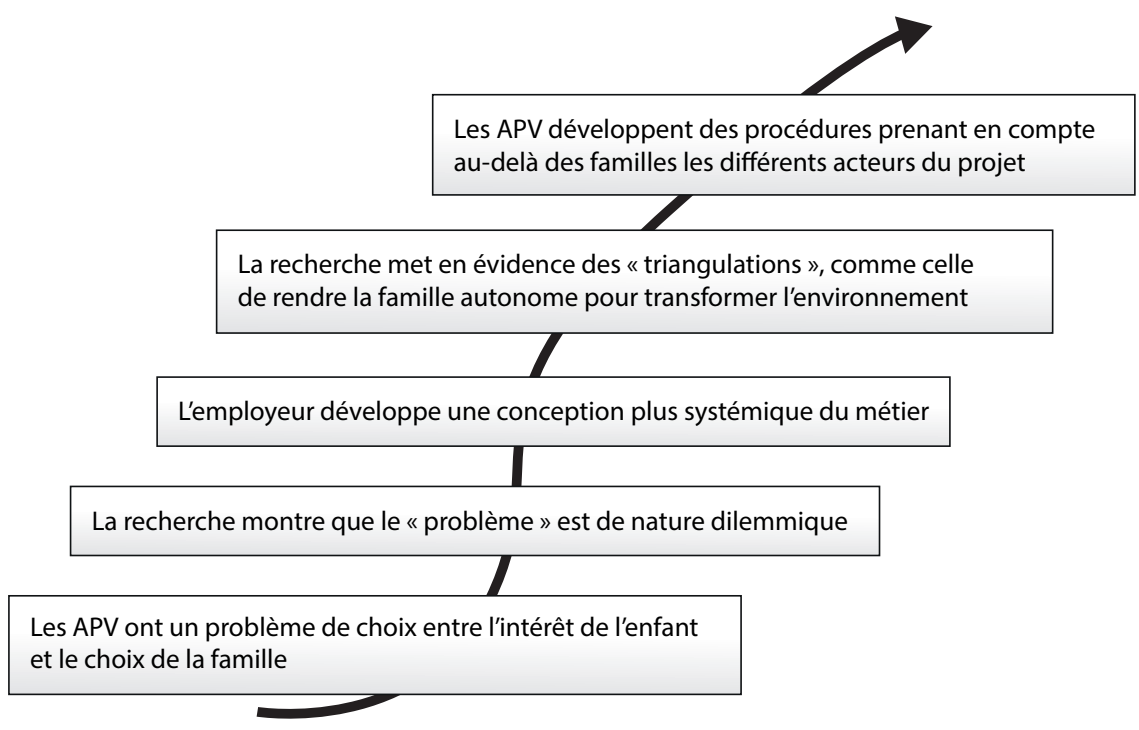

Lors d'une journée nationale, les APV soulèvent un problème relatif à leur accompagnement: doivent-ils suivre les choix de la famille ou l'intérêt du jeune? La recherche montre que ce qui fait problème est en réalité un dilemme de métier: accompagner le projet du jeune ou celui de ses parents et que pour se sortir de la question il faudrait peut-être reproblématiser avec la famille la situation pour attraper les choses autrement. Le problème n'est pas inhérent aux professionnels, il trouve, en effet, son origine dans la fiche de poste et les consignes des employeurs qui réitèrent que l'accompagnement doit porter sur la famille, mais dans la réalité des faits il y a parfois tension entre les deux projets (celui des parents et celui du jeune). La question est ensuite débattue avec les employeurs lors d'un comité technique où sont présentés les résultats intermédiaires du travail de recherche ce qui amène l'employeur à faire bouger ses représentations du métier et à l'envisager de manière plus systémique dans les interdépendances qui se nouent entre la famille, le jeune et le milieu. Creusant l'observation de ces mouvements, la recherche montre que le travail d'accompagnement peut s'ouvrir non seulement aux parents, mais à la 
famille élargie (notamment la fratrie), et demande aussi de prendre en compte l'évolution du milieu.

Ainsi, ce qui permet au métier de bouger, au-delà de l'engagement des professionnels et de leurs employeurs, ce sont d'une part les outils de la recherche, qui rendent le métier visible et lisible, et, d'autre part, des conditions sociales particulières d'échanges et de controverses qui permettent une analyse conjointe du réel et des interactions systémiques à l'œuvre entre professionnels, acteurs de la recherche et employeurs. Le dispositif mis en place " horizontalise " la lecture du métier ${ }^{5}$ et le positionne dans une situation d'intermétiers. Le travail commun est ainsi situé à la marge et au carrefour de différents métiers, c'est cet espace professionnel relativement nouveau qu'il nous a été permis d'observer et que nous nommons une situation d'intermétiers.

\section{DISCUSSION : \\ DES CHANGEMENTS DE PARADIGMES PARTAGÉS ENTRE PROFESSIONNELS DE L'ACCOMPAGNEMENT, DE LA RECHERCHE ET DE LA FORMATION}

\section{Des points d'appui théoriques modélisant le travail en situation d'intermétiers : la dimension épistémique du travail de recherche}

La recherche dont nous venons de rendre compte montre qu'agir en situation d'intermétiers c'est-à-dire aux carrefours d'une pluralité de métiers et faire évoluer cet espace professionnel est une dimension majeure du travail d'APV. Dans cette phase de construction du métier, nous pensons que l'accompagnement des professionnels par une recherche impliquée a été une condition de mise en place de cet espace d'intermétiers. Modéliser cette situation de travail en intermétiers est un vecteur de formation et de transformation des pratiques nécessaire pour ne pas réduire la question de la formation au seul compagnonnage ou à une construction solitaire de l'expérience sur le tas. Trois concepts permettent à nos yeux de théoriser la notion d'intermétiers, celui de "monde " emprunté à Béguin (2004) qui conceptualise la notion de point de vue au sens de là où chacun observe la situation commune. Cette observation est empreinte des valeurs, codes, normes de métiers, intérêts ou réglementation de chacun et fait particulièrement sens dans les situations d'intermétiers entre l'école, les familles, le secteur médico-social et la santé. La diversité de points de vue traduit le monde professionnel de chacun des acteurs engagés dans la situation. Ce faisant, le point de vue des uns entre en tension avec celui des autres à la fois dans la perception, mais aussi l'analyse et le traitement de la situation commune. Reconnaître ces tensions, les considérer comme légitimes est un premier pas vers l'autre avant que ne s'engagent des ajustements entre les mondes. Le second concept modélisant est celui d'espace d'intermétiers qui lui

5. Pour un développement de cet aspect le lecteur peut consulter Mérini, C., Thomazet, S., et Bélanger, J. (2018). La fabrication d'un métier, des « traductions " réciproques entre la recherche, les professionnels et les employeurs. Recherches Qualitatives "La fabrique interactive des analyses qualitatives " sous la direction de Joëlle Morissette et Annie Malo, 37(2), 105-127. doi:10.7202/1052110ar 
traduit l'existence d'un périmètre d'action partagé dans lequel s'exerce une activité professionnelle commune. Même s'il engage pleinement les familles, nous insistons tout d'abord sur la dimension professionnelle de cet espace, car il nécessite des compétences et des règles d'action spécifiques, celles de la collaboration (et non celles du métier d'origine) et qui peuvent être l'objet d'une formation. Cet espace est un territoire commun au sens où l'entend Moine (2014) c'est-à-dire un espace " habité " d'une certaine manière et fondé sur une organisation créatrice qui fait expérience commune. Espace et temps sont dans l'action intimement liés, la durée de l'action partagée est le temps du vécu commun. Cette durée de l'action signifie invention, création de formes, élaboration continue (Bergson, 1941/2009). Le rapport au temps de chacun des métiers est différent et soulève des tensions de temporalité qui vont devoir s'ajuster dans l'expérience grâce une organisation spécifique du travail et au partage de valeurs. L'organisation du travail commun se spatialise et se matérialise au travers de modes de coordination spécifiques (Mintzberg, 1982) permettant à chacun d'habiter l'espace commun. La conscience d'agir ensemble s'intellectualise au travers de points de réification permettant la circulation d'objets frontières (ce sera notre troisième concept) comme l'ont identifié une série d'auteurs (Star et Griesemer, 1989; Trompette et Vinck, 2009; Waitoller et Kozleski, 2013). Les objets frontières (cahiers de liaison, documents collaboratifs, concepts, valeurs ou notions partagées...) utilisés par des professionnels de plusieurs métiers peuvent ainsi contribuer à matérialiser l'agir ensemble.

Ainsi, échanges formels, informels ou formalisés vont permettre à ces points de réification de trouver des solutions partagées, d'échanger des outils ou des informations, de créer de nouveaux modes d'action, de les coordonner, etc. En retour "plus la conscience s'intellectualise plus la matière se spatialise " (Bergson, 1941/2009, p. 190) et cet espace d'intermétiers va devenir de plus en plus visible et, espérons-le, favoriser les processus inclusifs d'accessibilité. La présence de moments de négociations (Strauss, 1992) fondant les ajustements entre partenaires est pour nous un marqueur d'ouverture interprofessionnelle permettant de construire de nouvelles réponses à des situations résistantes, mais c'est aussi le signe d'un partage de responsabilité dans l'idée de rendre le milieu accessible aux personnes à besoins particuliers. Cette ouverture interprofessionnelle n'est pas sans rencontrer une série d'obstacles que nous présentons ci-après.

\section{Les situations d'intermétiers, des obstacles en tapinois, des compétences à développer}

Si dans les années 1970 le terme de participation était très employé, très vite la notion de partenariat s'est imposée dans l'espoir d'accélérer les prises de décision (Mérini, 2006). Au fil du temps, ces situations partenariales se sont multipliées, à propos d'objets très variés: la santé, les relations internationales, les pratiques artistiques, etc. (Zay, 1994). Du concept de partenariat à celui de situations d'intermétiers c'est pour nous cinquante ans de tentatives de réorganisation du travail qui posent la question de la fonction de coordination. Travailler en intermétiers c'est travailler dans les interstices, aux carrefours de différentes logiques et d'intérêts. Construire du commun pour agir ensemble 


\section{DOSSIER}

nécessite de s'éloigner des savoirs en jeu dans le métier d'origine, cette déprofessionnalisation (Wittorski, 2016) traduit une reprofessionnalisation liée à l'agir ensemble et à la collaboration.

Cette reprofessionnalisation n'est pas toujours présente dans les nouvelles fonctions de coordinateur de parcours ou de référent, lorsque celles-ci s'installent dans les interstices d'anciens métiers ou sur des modes de coordination bureaucratiques (Mintzberg, 2003/1986). À l'inverse de la coordination attendue, ces fonctions rendent les négociations impossibles et les décisions se prennent sous influences. Le travail en situation d'intermétiers possède des caractéristiques fortes. Situé à la marge de chacun des métiers, le travail est bien souvent transparent aux yeux mêmes des professionnels et donc difficile à faire valoir (Thomazet, Mérini et Gaime, 2014). Cette reconnaissance est aussi embarrassée du fait que les mondes, historiquement dissociés, ont construit des rapports asymétriques entre eux (école, famille, médico-social et sanitaire) et entre les acteurs (entre le médecin et les professions paramédicales par exemple) (Thomazet et Mérini, 2018). Par exemple gérer les relations de surplombance qui s'instaurent plus ou moins silencieusement lors des négociations, apprendre à les débusquer pour horizontaliser les échanges est une compétence à construire pour un professionnel de l'intermétiers. Dans un autre sens, les compétences d'intermétiers sont susceptibles de constituer des obstacles. C'est en effet, un espace professionnel d'action aux frontières organisationnelles faiblement formalisées, à périmètre variable et à partager (Maubant et Leclerc, 2008) selon les territoires et les cas traités. Son existence est sporadique et a vocation à s'éteindre une fois le problème résolu, elle ne peut donc être définitivement instituée comme l'ont été certaines organisations chargées d'accompagner les personnes comme les Maisons départementales des personnes handicapées (MDPH) par exemple. Notons enfin que le développement des métiers de l'intermétiers fera apparaître certaines difficultés, par exemple, la forte adaptation au milieu est potentiellement source d'inégalités entre les territoires.

\section{CONCLUSION}

Nous soutenons qu'aller vers une société inclusive est une responsabilité partagée et nous interrogeons dans ce texte les conséquences collectives du changement de paradigme qui peut nous y conduire. C'est l'occasion de nous interroger sur la place de la recherche, comme outil de compréhension des situations complexes et de formation des professionnels. Nous l'avons fait à partir d'une recherche intervention accompagnant l'émergence d'un nouveau métier, celui d'APV. Une démarche équivalente pourrait être menée pour accompagner la transformation des métiers des différents secteurs et l'émergence de nouveaux métiers de l'intermétiers. Les formations inter et l'accompagnement des collectifs d'intermétiers par une posture de recherche située au plus près de l'activité des professionnels nous semblent être des vecteurs de transformation de paradigme prometteurs. À cette condition le travail collectif pourra faciliter la construction d'une société plus accessible et constituer des pistes de formation novatrices. 


\section{Références}

Akrich, M., Callon, M., et Latour, B. (1988). À quoi tient le succès des innovations? 1: I'art de l'intéressement. Gérer et comprendre. Annales des mines, 11, 4-17.

Albero, B. (2010). De l'idéel au vécu: le dispositif confronté à ses pratiques. In B. Albero et N. Poteaux (dir.), Enjeux et dilemmes de l'autonomie. Une expérience d'autoformation à I'université. Étude de cas (pp. 67-94). Paris: MSH.

Bakhtine, M. (1978). Esthétique et théorie du roman. Paris: Gallimard.

Beguin, P. (2004). Mondes, monde commun et versions des mondes. Bulletin de psychologie, 57(469), 45-48.

Bergson, H. (1941/2009). L'évolution créatrice. Paris: PUF.

Bronfenbrenner, U. (1979). The ecology of human development: Experiments by nature and design. Cambridge, MA: Harvard University Press.

Callon, M. (1986). Éléments pour une sociologie de la traduction. L'année sociologique, 36, 169-208.

Caroly, S., et Clot, Y. (2004). Du travail collectif au collectif de travail. Des conditions de développement des stratégies d'expérience. Comparaison de deux bureaux. Formation et Emploi, 88, 43-55.

Cieslik, M., Legendre, M., de Decker, L., et Berrut, G. (2013). Bed-manager ou coordonnateur de parcours de soins. Gériatrie et psychologie neuropsychiatrie du vieillissement, 11(4), 337-338.

Clot, Y. (1999). La fonction psychologique du travail. Paris: Puf.

Clot, Y., Faïta, D., Fernandez, G., et Scheller, L. (2001). Entretiens en autoconfrontation croisée: une méthode en clinique de l'activité. Éducation permanente, 146, 17-25.

Ebersold, S. (2012). Parcours de scolarisation et coopération: enjeux conceptuels et méthodologiques. La nouvelle revue de l'adaptation et de la scolarisation, 57, 55-64.

Ebersold, S., Plaisance, E., et Zander, C. (2016). École inclusive pour les élèves en situation de handicap. Accessibilité, réussite scolaire et parcours individuels. [Rapport de recherche].

Gardou, C. (2012). La société inclusive, parlons-en! : il n'y a pas de vie minuscule. Toulouse: Érès.

Hill, N. E., \& Taylor, L. C. (2004). Parental school involvement and children's academic achievement: Pragmatics and issues. Current directions in psychological science, 13(4), 161-164.

Kalubi, J.-C., Detraux, J.-J., et Larivée, S. (2006). Introduction: Participation des familles en contexte d'inclusion sociale: une contribution en faveur de la bientraitance des élèves. Revue des sciences de l'éducation, 32(3), 517-524.

Kuhn, T. (1970). La structure des révolutions scientifiques. Paris: Flamarion.

Le Laidier, S. (2018). Note d'information N¹8-26 - Les enseignants accueillant des élèves en situation de handicap à l'école. Ministère de l'éducation nationale, de l'enseignement supérieur et de la recherche: <http://cache.media.education. gouv.fr/file/2018/39/8/depp-ni-2018-18-26-enseignants-accueillant-eleves-situation-de-handicap-ecole_1019398.pdts 


\section{DOSSIER}

Lesain-Delabarre, J.-M. (2012). Penser la coopération entre parents d'enfants handicapés et enseignants: un défi. La nouvelle revue de l'adaptation et de la scolarisation, 57, 79-92.

Maubant, P., et Leclerc, C. (2008). Le partenariat famille-école: à la recherche de I'improbable partenariat école-famille; origines d'un malentendu. In G. Pithon, C. Asdih et S.-J. Larivée (dir.), Construire une communauté éducative. Un partenariat famille-école-association (pp. 23-36). Bruxelles: De Boeck.

Mérini, C. (2006). Le partenariat en formation: de la modélisation à une application. Paris: L'Harmattan.

Mérini, C., et Bélanger, J. (à paraître). Pour une critique de l'observation des pratiques collaboratives. In C. Mérini, J.-F. Marcel et T. Piot (dir.), L'observation des pratiques collaboratives dans les métiers de l'interaction humaine: des pratiques pluri-adressées. Mont-Saint-Aignan: PUHR.

Mérini, C., et Ponte, P. (2008). La recherche-intervention comme mode d'approche des pratiques. Savoirs, 1(16), 77-95.

Mérini, C., Thomazet, S., et Bélanger, J. (2018). La fabrication d'un métier, des "traductions" réciproques entre la recherche, les professionnels et les employeurs. Recherches Qualitatives "La fabrique interactive des analyses qualitatives" sous la direction de Joëlle Morissette et Annie Malo, 37(2), 105-127. doi:10.7202/1052110ar

Mintzberg, H. (1982). Structure et dynamique des organisations. Paris: Éditions d'Organisation.

Mintzberg, H. (2003/1986). Le pouvoir dans les organisations. Paris: Éditions d'Organisation.

Moine, A. (2014, Mars). Le territoire comme un outil de transdisciplinarité vers des diagnostics partagés. Communication présentée au $2^{\mathrm{e}}$ colloque international du CIST - Fronts et frontières des sciences du territoire, Paris, France.

Morin, E. (1977). La méthode. 1. La nature de la nature. Paris: Seuil.

Nadeau, B. (1989). Le " case management " au carrefour de l'intervention clinique et communautaire. Santé mentale au Québec, 14(2), 51-59.

Prot, B., Mezza, J., Ouvrier-Bonnaz, R., Reille-Baudrin, E., et Vérillon, P. (2010). Les dilemmes d'activité. Pour une approche clinique des correspondances entre travail et formation professionnelle. Recherche et formation, 63, 63-76.

Saujat, F. (2010). Travail, formation et développement des professionnels de l'éducation. Note de synthèse pour I'HDR. Université d'Aix-Marseille I.

Star, S. L., \& Griesemer, J. R. (1989). Institutional ecology, translations' and boundary objects: Amateurs and professionals in Berkeley's Museum of Vertebrate Zoology, 1907-39. Social studies of science, 19(3), 387-420.

Strauss, A. (1992). La trame de la négociation: sociologie qualitative et interactionisme. Paris : L'Harmattan.

Thomazet, S. (2008). L'intégration a des limites, pas l'école inclusive. Revue des sciences de l'éducation, 34(1), 123-139.

Thomazet, S. (2017). Handicap et société inclusive. In F. Faberon et S. Urdician (dir.), Droit, culture et handicap (pp. 49-54). Aix-en-Provence: Presses universitaire d'Aix-Marseille. 
Thomazet, S., et Mérini, C. (2018). Analyse des asymétries du travail collectif dans un contexte d'école inclusive. In G. Rix et S. Thomazet (dir.), Interactions et dynamique des asymétries, Transverse, Soin/santé collection dirigée par Michaël Zicola (pp. 23-38).

Thomazet, S., Mérini, C., et Gaime, E. (2014). Travailler ensemble au service de tous les élèves. La nouvelle revue de l'adaptation et de la scolarisation, 65, 69-80.

Trompette, P., et Vinck, D. (2009). Retour sur la notion d'objet-frontière. Revue d'anthropologie des connaissances, 3(1), 5-27.

Veyrunes, P. (2011). Formats pédagogiques et configuration de l'activité collective à l'école primaire. (HDR), Université de Toulouse 2, Toulouse.

Vygotski, L. S. (1925/1994). Le problème de la conscience dans la psychologie du comportement (F. Sève, Trad.). Société française, 50, 35-47.

Waitoller, F., R., \& Kozleski, E., B. (2013). Working in boundary practices: identity development and learning in partnerships for inclusive education. Teaching and Teacher Education, 31, 35-45.

Wittorski, R. (2016). L'injonction à l'interdisciplinarité: un symptôme de (dé)professionnalisation. Forum, 148, 44-51.

Zay, D. (dir.) (1994). Enseignants et partenaires de l'école, démarches et instruments pour travailler ensemble. Paris / Bruxelles: INRP/ De Boeck. 